International scientific and technical conference

DOI: $10.34185 / 1991-7848 . i t m m .2020 .01 .018$

\title{
КВАДРАТИЧНЫЕ ОПТИМИЗАЦИОННЫЕ МОДЕЛИ И МЕТОДЫ
}

Косолап А.И. докт. физ.-мат. наук, проф.,

Украинский государственный химико-технологический университет, Украина

Ключевые слова: КВАДРАТИЧНЫЕ МОДЕЛИ, ОПТИМИЗАЦИЯ, МУЛЬТИМОДАЛЬНЫЕ ЗАДАЧИ, МЕТОД ТОЧНОЙ КВАДРАТИЧНОЙ РЕГУЛЯРИЗАЦИИ.

Введение. Фактически в любой сфере человеческой деятельности при проектировании сложных систем необходимо выбирать наилучшие решения. Это позволяет экономить ресурсы, время, деньги. Для построения математических моделей систем разработан инструментарий. Наиболее простыми являются линейные модели с непрерывными переменными число которых может быть достаточно большим. Разработаны эффективные методы, позволяющие находить оптимальные значения переменных в линейных моделях. Однако класс задач, которые можно описать линейными моделями, очень ограничен. Большинство проектируемых систем являются нелинейными. Для таких систем строятся нелинейные модели и используются методы решения нелинейных оптимизационных задач. Попытка аппроксимации нелинейных систем линейными в многомерном евклидовом пространстве является неэффективной. В большинстве случаев, сложные системы могут быть описаны квадратичными моделями. Если квадратичные модели сводятся к минимизации выпуклой функции на выпуклом множестве, то существуют эффективные методы для решения таких классов задач [1]. Однако систем с выпуклыми квадратичными моделями очень мало. В тоже время, общими квадратичными функциями можно описать значительное число сложных систем.

Рассмотрим линейные модели с булевыми переменными

$$
\min \left\{\mathrm{c}^{\mathrm{T}} \mathrm{x} \mid \mathrm{Ax} \leq \mathrm{b}, \mathrm{x}=0 \text { или } 1, \mathrm{x} \in \mathrm{E}^{\mathrm{n}}\right\},
$$

где $A$ - матрица $(m \times n)$, векторы $c, b$ заданы и необходимо найти $x$. Существует огромное число систем, которые могут быть описаны такими моделями. Очень легко такие модели преобразуются к квадратичным. Это достигается при помощи квадратичного ограничения 


$$
\sum_{i=1}^{m} \sum_{j=1}^{n}\left(1-x_{i j}\right) x_{i j} \leq 0,0 \leq x \leq 1
$$

Существуют модели на перестановках целых чисел

$$
\min \left\{c^{T} x \mid A x \leq b, x \in\{1, \ldots, n\}\right\} .
$$

Эти модели также сводятся к квадратичным

$$
\min \left\{c^{T} z \mid A z \leq b, \sum_{j=1}^{n} z_{i j}=i, i=1, \ldots, n, \sum_{i=1}^{n} \sum_{j=1}^{n} z_{i j}^{2} \geq \sum_{i=1}^{n} i^{2}, \sum_{j=1}^{n}\left(\sum_{i=1}^{n} x_{i j}\right) \leq \sum_{i=1}^{n} i_{i}^{2}\right\},
$$

где

$$
z_{j}=\sum_{i=1}^{n} z_{i j}, j=1, \ldots, n, z=\left(z_{1}, \ldots, z_{n}\right) .
$$

Аналогичное преобразование возможно и для дискретных значений переменных

$$
\min \left\{c^{T} x \mid A x \leq b, x \in\left\{u_{1}, \ldots, u_{m}\right\}\right\}
$$

Эта задача преобразуется к виду

$$
\min \left\{c^{T} z \mid A z \leq b, \sum_{j=1}^{n} z_{i j}=u_{i}, i=1, \ldots, m, \sum_{i=1}^{m} \sum_{j=1}^{n} z_{i j}^{2} \geq \sum_{i=1}^{n} u_{i}^{2}, \sum_{j=1}^{n}\left(\sum_{i=1}^{m} x_{i j}\right)^{2} \leq \sum_{i=1}^{m} u_{i}^{2}\right\} .
$$

Если модель описана с помощью многомерных полиномов, то посредством последовательной замены $z=x^{2}, z_{i j}=x_{i} x_{j}$ такая модель преобразуется к квадратичной.

Существует большой класс задач оптимального распределения дискретных ресурсов. Это задачи о назначениях или, например, задача распределения заданий в многопроцессорных системах

$$
\min \left\{\sum_{i=1}^{n}\left(\sum_{j=1}^{m} t_{j} x_{i j}\right)^{2} \mid \sum_{j=1}^{m} x_{i j}=1, i=1, \ldots, n, x=0 \vee 1\right\},
$$

где переменные принимают только булевы значения

$$
x_{i j}=\left\{\begin{array}{l}
1, \text { если } i \text {-е задание выполняется } j \text {-м процессором } \\
0, \text { в противном случае }
\end{array}\right.
$$

Эта задача также преобразуется к квадратичной

$$
\min \left\{\sum_{i=1}^{n}\left(\sum_{j=1}^{m} t_{j} x_{i j}\right)^{2} \mid \sum_{j=1}^{m} x_{i j}=t_{j}, i=1, \ldots, n, \sum_{i=1}^{n} \sum_{j=1}^{m} x_{i j}^{2} \geq \sum_{j=1}^{m} t_{j}^{2}\right\} \text {. }
$$

В общем случае, квадратичную задачу запишем в виде

$$
\min \left\{x^{T} A_{0} x+b_{0}^{T} x \mid x^{T} A_{i} x+b_{i}^{T} x+c_{i} \leq 0, i=1, \ldots, m\right\},
$$


International scientific and technical conference Information Technologies in Metallurgy and Machine building - ITMM 2020

где матрицы $A_{i}, \forall i$ симметричные, в которой необходимо найти $n$-мерный вектор х. Такая задача будет мультимодальной и NP-полной. Долгое время такие задачи практически не исследовались и только после разработки полуопределенной оптимизации ее начали применять для решения общих квадратичных задач [2].

Большинство алгоритмов оптимизации начинают поиск оптимальной точки с допустимой точки (возможно и с недопустимой точки) и на каждой итерации переходят в следующую точку с меньшим значением целевой функции. Если алгоритм приводит в точку локального минимума, то поиск завершается, так как любое перемещение с этой точки приводит к возрастанию целевой функции. Часто задачи имеют большое число локальных минимумов, например, $2^{n}$ или $n$ ! В таких случаях, локальные методы остановятся в окрестности начальной точки. Возникает проблема выхода из локального минимума. Классический анализ не может решить эту проблему, так как он оценивает значение функции только в окрестности точки. Поэтому для решения мультимодальных задач используют методы мультистарта, ветвей и границ, случайного поиска. Но эти методы не гарантируют получение точки глобального минимума особенно для задач большой размерности [3-4].

Мы предлагаем для решения задач (1) использовать метод точной квадратичной регуляризации. Квадратичная регуляризация позволяет преобразовать задачу (1) к следующей

$$
\begin{aligned}
& \max \left\{\|x\|^{2} \mid x^{T} A_{0} x+b_{0}^{T} x+s+(r-1)\|x\|^{2} \leq d,\right. \\
& \left.x^{T} A_{i} x+b_{i}^{T} x+c_{i}+r\|x\|^{2} \leq d, i=1, \ldots, m\right\}
\end{aligned}
$$

в которой необходимо найти максимум нормы вектора на выпуклом множестве. При таком преобразовании используются два параметра $s, r$ и две новых переменных $x_{n+1}, d$. В задаче (2) необходимо найти минимальное значение переменной $d$ и $x$, для которых выполниться условие $r\|x\|^{2}=d$. Это значение $d$ находим методом дихотомии.

Преобразованная задача (2) также мультимодальная, но решить ее значительно проще. Для того чтобы показать как этот метод выводит с точки локального экстремума, рассмотрим самый простой случай, когда выпуклое множество является отрезком $\max \left\{\|x\|^{2} \mid x \in[a, b]\right\}$. Будем предполагать, что 
Ministry of Education and Science of Ukraine

The National Metallurgical Academy of Ukraine, Dnipro, 17 - 19 March, 2020

решение задачи $\max \left\{e^{T} x \mid x \in[a, b]\right\}(e=(1, \ldots, 1))$ достигается в единственной точке. Задача максимума нормы вектора на отрезке мультимодальная, если решение выпуклой задачи $\min \left\{\|\left. x\right|^{2} \mid x \in[a, b]\right\}$ находится внутри отрезка и унимодальная, если ее решение лежит за пределами этого отрезка. Сместим отрезок вдоль биссектрисы положительного ортанта таким образом, чтобы точка минимума находилась за пределами отрезка. Тогда задача максимума станет унимодальной и если до смещения алгоритм остановился в точке локального максимума нормы вектора, то после смещения поиск будет продолжено в точку глобального максимума. Это справедливо и для более общего случая, когда отрезок заменить на многогранник. Если задача $\min \left\{e^{T} x \mid A x \leqslant b, x \geqslant 0\right\}$ имеет единственное решение в искомой точке, то существует такое смещение, что максимум нормы вектора на смещенном многограннике будет унимодальной задачей. Если же задача $\min \left\{\|x\|^{2} \mid A x \leqslant b, x \geqslant 0\right\}$ имеет неединственное решение, то ее решение находится на грани многогранника. В этом случае легко построить линейное преобразование пространства, после которого на данной грани задача будет иметь единственное решение.

Выводы. В работе показано, как многие сложные системы могут быть описаны квадратичными моделями. Для их решения предлагается использовать эффективный метод точной квадратичной регуляризации со смещением пространства. Численные эксперименты подтверждают эффективность данного метода для решения общих квадратичных задач.

\section{Литература}

1. Nocedal J. Numerical optimization / J. Nocedal, S.J. Wright. - Springer, 2006. - 685 p.

2. Ye Y. Semidefinite programming /Y. Ye. - Stanford University, 2003. - 161 p.

3. Horst R. Global Optimization: Deterministic Approaches. 3rd ed./ R. Horst, H. Tuy. Berlin: Springer-Verlag, 1996. - 727 p.

4. Kenneth V. P. Differential Evolution. A Practical Approach to Global Optimization / V.P. Kenneth, R.M. Storn,J. A. Lampinen. - Berlin, Heidelberg: Springer-Verlag, 2005. - 542 p. 


\section{QUADRATIC OPTIMIZATION MODELS AND METHODS}

\section{Kosolap Anatolii}

Abstract. In this paper we show how the complex systems are described by quadratic optimization models. We show how the problems with Boolean variables, the problems with discrete variables and permutations are transformed to the quadratic models. In general, quadratic optimization models are multimodal. We propose using the effective method of exact quadratic regularization to search for the variables of quadratic models. Quadratic regularization allows us to transform general quadratic problems to maximize the norm of a vector on a convex set. We show in which cases the displacement of a convex set along the bisector of a positive orthant allows us to transform the problem to unimodal. Numerical experiments confirm the effectiveness of the proposed method for solving general quadratic problems.

Keywords: QUADRATIC MODELS, OPTIMIZATION, MULTIMODAL PROBLEMS, EXACT QUADRATIC REGULARIZATION METHOD.

\section{References}

1. Nocedal J., Wright S.J. Numerical optimization. Springer, 2006. 685 p.

2. Ye Y. Semidefinite programming. Stanford University, 2003. 161 p.

3. Horst R., Tuy H. Global Optimization: Deterministic Approaches. 3rd ed. Berlin: SpringerVerlag, 1996. 727 p.

4. Kenneth V. P., Storn R. M., Lampinen J. A. Differential Evolution. A Practical Approach to Global Optimization. Berlin, Heidelberg: Springer-Verlag, 2005. 542 p. 